\title{
Bloch surface wave label-free and fluorescence platform for the detection of VEGF biomarker in biological matrices
}

Riccardo Rizzo ${ }^{\mathrm{a}}$, Maria Alvaro ${ }^{\mathrm{b}}$, Norbert Danz ${ }^{\mathrm{c}}$, Lucia Napione ${ }^{\mathrm{b}}$, Emiliano Descrovi ${ }^{\mathrm{a}}$, Stefan Schmieder $^{\mathrm{d}}$, Alberto Sinibaldi ${ }^{\mathrm{e}}$, Rona Chandrawati ${ }^{\mathrm{f}, \#}$, Subinoy Rana ${ }^{\mathrm{f}}$, Peter Munzert ${ }^{\mathrm{c}}$, Thomas Schubert $^{\mathrm{g}}$, Emmanuel Maillart ${ }^{\mathrm{h}}$, Aleksei Anopchenko, ${ }^{\mathrm{e},}$, Paola Rivolo ${ }^{\mathrm{a}}$, Alessandro Mascioletti ${ }^{\mathrm{i}}$, Frank Sonntag $^{\mathrm{d}}$, Molly M. Stevens ${ }^{\mathrm{f}}$, Federico Bussolino ${ }^{\mathrm{b}}$ and Francesco Michelotti ${ }^{\mathrm{e}}$

${ }^{a}$ Department of Applied Science and Technology, Politecnico di Torino, C.so Duca degli Abruzzi 24, 10129 Torino, Italy

b Laboratory of Vascular Oncology, Candiolo Cancer Institute - IRCCS, Candiolo, Italy. Department of Oncology, University of Torino, Candiolo, Italy.

${ }^{c}$ Fraunhofer Institute for Applied Optics and Precision Engineering IOF, Albert-Einstein-Str. 7, Jena 07745 , Germany

${ }^{\mathrm{d}}$ Fraunhofer Institute for Material and Beam Technology IWS Dresden, Dresden 01277, Germany

e Department of Basic and Applied Science for Engineering, Sapienza University of Rome, Via A. Scarpa 16, 00161 Rome, Italy

${ }^{\mathrm{f}}$ Department of Materials, Imperial College London, London SW7 2AZ, U.K.

g KDS Raderberger Gmb.H, Großröhrsdorf 01900, Germany

${ }^{\text {h }}$ HORIBA Scientific, Av.de la Vauve CS 45002 - 91120 Palaiseau, France

${ }^{\text {i } L A B O R ~ S r l, ~ V i a ~ G . ~ P e r o n i, ~} 00131$ Rome, Italy

\section{Corresponding authors:}

Francesco Michelotti: francesco.michelotti@uniroma1.it

Federico Bussolino: federico.bussolino@unito.it

\section{Present Addresses}

\# School of Chemical and Biomolecular Engineering, The University of Sydney, Sydney, NSW 2006, Australia.

$\S$ Department of Physics, Baylor University, One Bear Place \#97316, 76798-7316, Waco, Texas. 
Abstract: We report on the detection of an angiogenic molecule Vascular Endothelial Growth Factor (VEGF) in different biological matrices by means of a new integrated biosensing platform exploiting the properties of Bloch surface waves. The new platform takes advantage of a tandem configuration, in which both label-free and enhanced fluorescence detection are implemented. Specifically designed one dimensional photonic crystals were deposited directly on disposable and low cost plastic biochips. A direct sandwich immunoassay was used to detect VEGF in buffer, cell culture supernatant and human plasma at low concentration $(\mathrm{ng} / \mathrm{mL})$. The platform enabled the detection of VEGF in all three matrices with high resolution, fast turnaround time (30 minutes) and in close agreement with the results of enzyme linked immunosorbent assays.

Keywords: biosensor platform, Bloch surface waves, label-free optical sensor, enhanced fluorescence, tumor biomarker, complex matrices.

\section{Introduction}

Quantitative detection of cancer biomarkers is a powerful tool to diagnose cancers in early stages (Pepe et al., 2001). Furthermore, cancer biomarkers can be used for noninvasive monitoring of treatment response in individual patients. Among biomarkers, vascular endothelial growth factor (VEGF) is an angiogenic inducer that plays a key role in cancer progression and dissemination as well as in tumor angiogenesis (Ferrara, 2010).

The enzyme-linked immunosorbent assay (ELISA) is considered the preferred technique for measuring a biomarkers' concentration in plasma samples. However, conventional ELISA tests are time-consuming, require expert personnel and specific laboratory equipment (Tighe et al., 2015). The development of a sensitive, compact and easy-to-use platform for real-time detection of biomarkers would therefore present a major advancement in both clinical and research field.

Optical biosensing techniques provide a viable alternative to ELISA for the detection of different kinds of biomolecules including tumor biomarkers (Tan et al., 2008; Ulber et al., 2003) and are usually classified as either fluorescence or label-free, depending on the use or not of fluorescent labels (Walker, 2009). Both families of techniques show advantages and drawbacks. Label-free techniques, such as surface plasmon resonance (SPR), are generally used to investigate in real-time the kinetics of surface-binding events, providing quantitative information on specific biomolecular binding constants (Dey et al., 2012; Guo, 2012; Sikarwar et al., 2017; Ulber et al., 2003). While the latter is a unique and valuable feature of label-free methods, resolution is usually lower compared to 
the fluorescence-based methods (Mathias et al., 2007). For such a reason, fluorescence schemes exploiting evanescent waves, featuring strong field localization and intensity enhancement at the surface of interest, have been employed (Anopchenko et al., 2016; Cooper, 2002; Descrovi et al., 2013; Fan et al., 2008; Ligler and Taitt, 2008; Zhao et al., 2010). Among evanescent wave sensors, those based on Bloch surface waves (BSW) sustained by dielectric one dimensional photonic crystals (1DPC) (Yeh et al., 1977) have been demonstrated as an effective alternative to SPR for label-free optical biosensing (Konopsky et al., 2013; Rivolo et al., 2012; Rizzo et al., 2014; Shinn and Robertson, 2005; Sinibaldi et al., 2015a, 2015b). The direct comparison of the performance of BSW with SPR biosensors showed that evanescent waves on all-dielectric structures can outperform their metallic analogue (Sinibaldi et al., 2012). Furthermore, BSW were used to enhance the fluorescence emission near 1DPC surfaces (Angelini et al., 2014, 2013; Badugu et al., 2013; Descrovi et al., 2010; Ray et al., 2015; Ricciardi et al., 2015). It is therefore clear that integrating both label-free and fluorescence detection leads to an improvement of the performance of biosensing platforms, as shown also with photonic crystal biochips (Inan et al., 2017) and resonant waveguide gratings (Fang et al., 2006).

Although encouraging, all BSW coupled fluorescence experiments were conducted with biomarkers spiked in simple matrices such as Dulbecco phosphate buffered saline (D-PBS). In order to obtain clinically relevant results it is essential to perform the biomarker detection in more complex matrices, either in human plasma or other biological fluids.

Recently, the use of BSW for fluorescence and label-free (tandem) detection of the ERBB2 breast cancer biomarkers in cell lysates was reported (A. Sinibaldi et al., 2017). The results were obtained by means of an extended laboratory optical setup and 1DPC deposited on microscope slides.

Here, we report for the first time on the use of a much more compact point-of-care platform, operating in such a tandem configuration (Danz et al., 2015), to detect VEGF in several different biological matrices with increasing level of complexities. The platform makes use of BSW on disposable and low cost 1DPC plastic biochips, with a micro-patterned immobilization that provides on chip test and reference spots. The lowest level of sample complexity was represented by solutions of VEGF (recombinant protein) dissolved in D-PBS. Complexity was increased by assaying cell culture supernatants, in which small amounts of other biomolecules are released in addition to VEGF. Finally, the highest level of sample complexity was provided by VEGF spiked in human plasma. The presence of VEGF in cell supernatant and blood samples was quantified with ELISA kits and the results were compared with those obtained with our biosensing platform. The experiments show that our platform can effectively detect the same biomarker in different matrices 
with high specificity. Furthermore, use of the tandem configuration effectively improves the performance by reducing the limit of detection (LoD).

\section{Materials and Methods}

\subsection{Materials and biological samples}

Sulfuric acid (95-98\%), hydrogen peroxide (30\% in $\mathrm{H}_{2} \mathrm{O}$ ), (3-aminopropyl)triethoxysilane (APTES, 99\%), ethanol (99.8\%), glutaraldehyde solution (grade I, $50 \%$ in $\mathrm{H}_{2} \mathrm{O}$ ), sodium bicarbonate (99.7\%), sodium cyanoborohydride (95\%), hydrogen chloride (2M), glycine (98.5\%), bovine serum albumin (BSA, 98\%), fetal bovine serum (FBS) and Dulbecco's Phosphate Buffered Saline 1X (DPBS) were purchased from Sigma-Aldrich. Dulbecco's Modified Eagle Medium (DMEM) was purchased from Lonza. NeutrAvidin Protein DyLight 650 (1 mg/mL) was purchased from Thermo Fisher Scientific. Anti-human VEGF capture antibody (Ab-VEGF), recombinant human VEGF 165 protein $(97 \%)$ (VEGF), biotinylated anti-human VEGF detection antibody (Ab-VEGF*), antihuman Angiopoietin-1 capture antibody (Ab-Ang1), and Human VEGF Quantikine ELISA Kit were purchased from R\&D System. Water was purified by means of a Merck-Millipore deionizer. All materials were used as purchased.

We prepared the lowest complexity samples by diluting recombinant VEGF in D-PBS.

Samples characterized by an intermediate level of complexity were prepared from cells releasing VEGF in supernatants. In particular we used wild-type and VEGF $_{165}$-overexpressing MCF-7 cells, which are an extensively used human breast adenocarcinoma line (Comşa et al., 2015). Cells were seeded in culture dishes at the density of $3.0 \times 10^{5}$ cells $/ \mathrm{cm}^{2}$ with DMEM containing $10 \%$ FBS. Serum-free medium was conditioned for $48 \mathrm{~h}$ by confluent cultures. After collection, cell supernatants were cleaned by centrifugation $\left(1500 \mathrm{rpm}\right.$ for $10^{\prime}$ at $\left.4^{\circ} \mathrm{C}\right)$ to remove particles. Samples were aliquoted and stored at $-20^{\circ} \mathrm{C}$.

Human plasma samples from blood of cancer patients were collected at the Institute for Cancer Research and Treatment in Candiolo (Italy), in accordance with the protocol approved by the Ethical Committees of the Institute and of the Piedmont Region (Italy). Plasma was prepared by centrifugation of the whole blood contained in the test tube with EDTA as anticoagulant; after centrifugation the corpuscolate fraction was discarded and the supernatant (i.e. plasma) was collected, aliquoted and stored at $-80{ }^{\circ} \mathrm{C}$. 


\subsection{Photonic crystal biochips}

In Fig. 1(a), we show a photograph of the disposable plastic biochip together with its complementary microfluidic cover. In order to provide a disposable and low cost sensor unit, we deposited the 1DPC directly on a molded plastic (TOPAS $®$ ) substrate with prismatic windows. Fig. 1(b) shows a rendering of the sensing section of the biochip with the 1DPC stack (not to scale). The biochip allows the excitation of a BSW when illuminated from the bottom through the windows in the Kretschmann-Raether total internal reflection (TIR) configuration, similar to SPR. After chemical functionalization, the two-components plastic cover, with a soft polymer layer defining a $800 \mu \mathrm{m}$ wide fluidic channel and guaranteed sealing, is clicked on top of the chip and permits the injection of fluids for the analysis.

The 1DPC were deposited directly on the plastic substrates by plasma ion-assisted evaporation under high vacuum conditions (Leybold Optics, APS904). Such 1DPC are dielectric stacks with alternate high and low refractive index layers. The low refractive index material is $\mathrm{SiO}_{2}$ (silica), $\mathrm{n}_{\mathrm{SiO} 2}=1.474+\mathrm{i} 5 \mathrm{E}-6$, while the two materials $\mathrm{Ta}_{2} \mathrm{O}_{5}$ (tantala), $\mathrm{n}_{\mathrm{Ta} 2 \mathrm{O} 5}=2.160+\mathrm{i} 5 \mathrm{E}-5$, and $\mathrm{TiO}_{2}$ (titania), $\mathrm{n}_{\mathrm{TiO} 2}=2.28+\mathrm{i} 1.8 \mathrm{E}-3$ are used as high refractive index layers. The complex refractive indices of the materials were determined by ellipsometry on test multilayers sustaining BSW at the selected wavelength of $\lambda=670 \mathrm{~nm}$. In order to promote stack adhesion to the plastic substrate we deposited a first silica layer of $275 \mathrm{~nm}$. In the 1DPC, a periodic stack made out of two tantala/silica $\left(\mathrm{d}_{\mathrm{Ta} 2 \mathrm{O} 5}=120 \mathrm{~nm}, \mathrm{~d}_{\mathrm{SiO} 2}=275 \mathrm{~nm}\right)$ bilayers is topped by a thin bilayer made out of titania/silica $\left(\mathrm{d}_{\mathrm{TiO} 2}=\mathrm{d}_{\mathrm{SiO} 2}=20 \mathrm{~nm}\right)$. The $1 \mathrm{DPC}$ was purposely designed to operate in water and to exhibit a high label-free sensitivity together with an extraordinary enhancement of the out-coupled fluorescence (Rizzo et al., 2014).
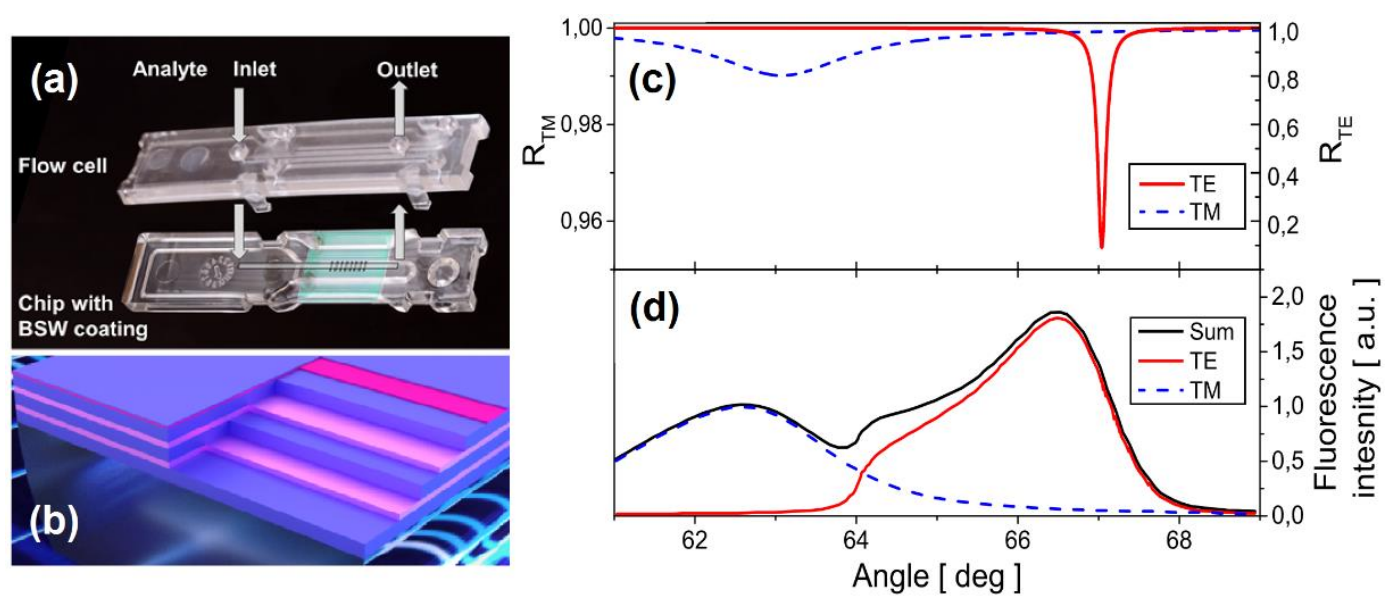
Fig. 1: (a) Photo of a TOPAS ${ }^{\circledR}$ biochip, with the 1DPC (green appearance), and its polymeric microfluidic cover. (b) Rendered image of a cross section of the 1DPC, with different layers deposited on top of the biochip (not to scale). (c) Calculated angular reflectivity profile for TE and TM polarized light at $\lambda=670 \mathrm{~nm}$, (d) simulated intensity distribution of the fluorophore DyLight 650 over an 8 deg angular range for TE, TM, and Sum emission polarization.

In Fig. 1(c) we show the TIR angular spectrum of the biochips according to the design. The curves were calculated for both TE and TM polarized incident light at $\lambda=670 \mathrm{~nm}$, when the external medium is water, $\mathrm{n}_{\mathrm{H} 2 \mathrm{O}}=1.330$. The dips in the spectrum indicate that a BSW is excited at about $67 \mathrm{deg}$ when using the TE polarization and at about $63 \mathrm{deg}$ in the TM case. Given its reduced width and large depth, the TE dip is used for label-free operation. On the other hand, both TE and TM BSW are involved in the fluorescence operation.

\subsection{Optical setup}

Fig. 2 illustrates the optical excitation and detection system operating in the Kretschmann-Raether configuration and making use of the biochips described above. A collimated, TE polarized beam of a $670 \mathrm{~nm}$ laser diode is focused by a cylindrical lens (CL1) into the polymer chip, thus illuminating a line at the sensor surface. The reflected light is angularly imaged onto the CCD image sensor (APOGEE Ascent with Sony ICX814) by the cylindrical lens CL2. Therefore, each row of the CCD image can be assigned to one angular component of the reflected light in order to measure the angular reflectivity curve plotted in Fig. 1(c). As a result, an angular range of 2.9 deg can be analyzed along the long dimension of the CCD (3388 pixel). In the label-free experiments reported below, we further restricted the angular range around the resonance to about $1 \mathrm{deg}$ (1100 pixel), to reduce the memory occupation and increase the CCD frame rate.

More than 20 spots can be arranged along the illuminated line at the sensor surface. These are imaged by a cylindrical optical system (CL3 and CL4) onto the columns of the CCD (2712 pixel) in order to allow one for analyzing all spots independently.

As already mentioned, the excitation of a BSW leads to an enhancement of the electric field at the interface between the 1DPC surface and the external medium. We use such enhancement to excite fluorophores at the stack surface by a $635 \mathrm{~nm}$ laser diode and to boost their emission into the direction of the substrate. The resonance for fluorescence excitation at $635 \mathrm{~nm}$ is angularly shifted 
and is as narrow as the resonance used for label-free sensing. Its angular position will also shift during the experiments when molecules bind onto the sensor surface (the same effect as the one exploited for label-free sensing). For this reason the angle of fluorescence excitation needs to be scanned in order to find the correct excitation angle for exploiting surface wave enhanced fluorescence excitation. Here, the collimated beam of the $635 \mathrm{~nm}$ laser diode is focused by a $100 \mathrm{~mm}$ focal length cylindrical lens into the sensor (green rays in Fig. 2) thus illuminating the same region that is illuminated in the label-free mode. The dichroic splitter SP combines the different wavelengths. Laterally shifting the collimated laser beam keeps the illuminated position constant but changes the angle of excitation.

The resulting fluorescence emission is angularly dispersed due to the dispersion of the BSW thin film stack as shown in Fig. 1(d), which presents the simulated intensity distribution of an isotropically oriented ensemble of fluorophores DyLight 650 over an 8 deg angular range. The angular emission spectrum (water environment) is characterized by two broad peaks resulting from coupling of fluorophores' emission to either TE or TM polarized BSW. The dichroic splitter SP transmits the emitted fluorescence that is imaged angularly and laterally by the same optical system onto the same detector as in the label-free case.

The angular range of $2.9 \mathrm{deg}$ available in the label-free mode is insufficient to collect all fluorescence energy emitted into the $8 \mathrm{deg}$ angular range. For this reason, the collection optics is equipped with a cylindrical system ("zoom” in Fig. 2 and Fig. S3, Supplementary Material), which can be inserted along the optical path when the platform is switched from the label-free to the fluorescence mode. This increases the observed angular range, thus enabling to collect almost all fluorescence, reducing the angular resolution that is not required in the fluorescence mode. Upon switching, the label-free/fluorescence lasers are switched off/on, and the CCD integration time is automatically adapted to operate in a linear range. 


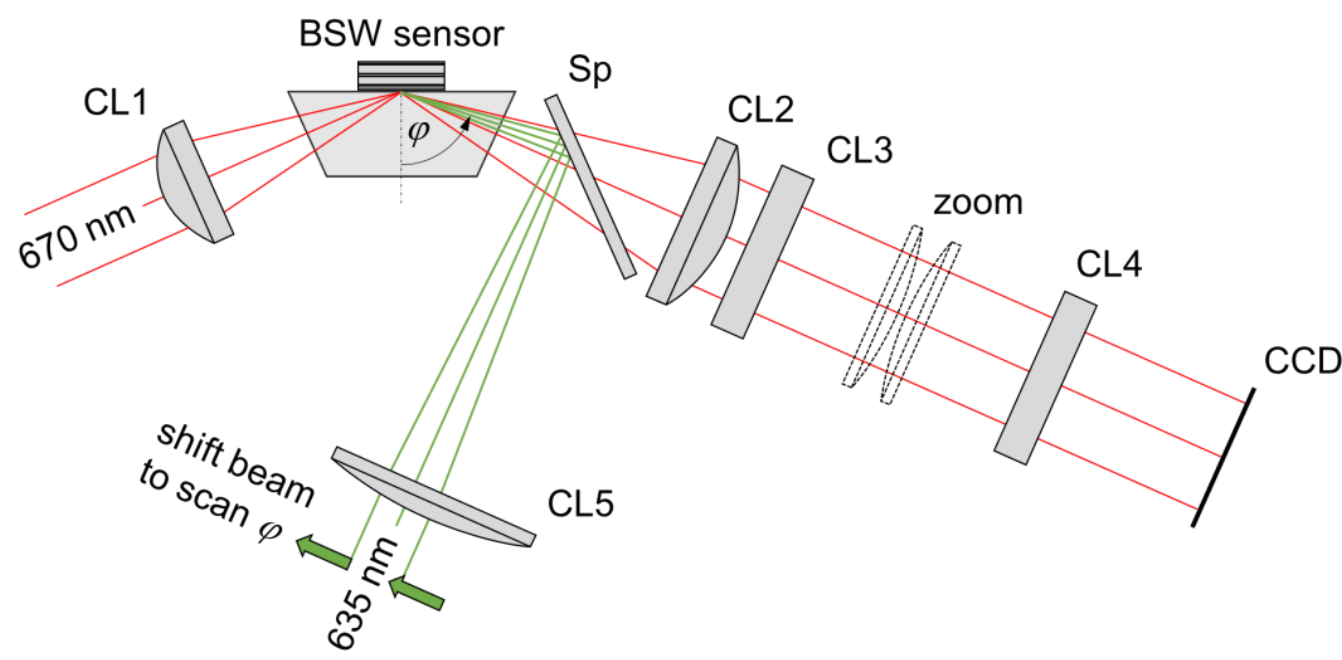

Fig. 2: A sketch illustrating the optical system without plotting laser diode collimation systems. Label-free and fluorescence emission is shown in red while fluorescence excitation is illustrated in green. See text for details.

\subsection{Surface functionalization and antibody immobilization}

In order to perform immunoassays on the 1DPC biochips, their surface was chemically functionalized to allow an effective conjugation of capture antibodies. Among the various surface modification techniques, we made use of the derivatization of the $\mathrm{SiO}_{2}$ surface by means of a selfassembled organosilane (APTES) layer (Fiorilli et al., 2008). Glutaraldehyde (GAH) was used as a homo-bifunctional cross-linker between the primary amines of APTES and the amine groups of the antibodies/proteins to be bound at the surface. Details on the development and final realization of the full functionalization protocol by APTES/GAH can be found in the Supplementary Material (Fig. S4 and Fig. S5, Supplementary Material). Briefly, the 1DPC surfaces were first cleaned with piranha solution $\left(\mathrm{H}_{2} \mathrm{SO}_{4}: \mathrm{H}_{2} \mathrm{O}_{2}=3: 1\right)$ for $10 \mathrm{~min}$, and washed thoroughly with de-ionized water and ethanol, followed by drying with $\mathrm{N}_{2}$. The clean biochips were immersed in an APTES solution (2\% $\mathrm{v} / \mathrm{v})$ in a mixture of ethanol/water $(95: 5 \mathrm{v} / \mathrm{v})$ for 1 hour at room temperature. The chips were then washed in pure ethanol, dried with $\mathrm{N}_{2}$, and baked on a hot plate at $110^{\circ} \mathrm{C}$ for 1 hour. The APTESmodified chips were reacted with $1 \%$ (v/v) glutaraldehyde in $100 \mathrm{mM}$ sodium bicarbonate buffer $(\mathrm{pH} 8.5)$ in the presence of $0.1 \mathrm{mM}$ sodium cyanoborohydride for 1 hour at room temperature, followed by washing in de-ionized water. The successive protein immobilization steps were always carried out within one day from the APTES/GAH step, for biochips stored dry at $4{ }^{\circ} \mathrm{C}$. 
In order to discriminate between specific and non-specific binding we incubated and immobilized different antibodies in different regions of the functionalized 1DPC surface. For this purpose, we used a polydimethylsiloxane (PDMS) functionalization microfluidic tool (Supplementary Material, Fig. S7, Supplementary Material), consisting of five parallel flow channels. The tool is pressed on the surface of the GAH activated biochip and different solutions are injected and incubated in each channel separately. Here, we used the tool to immobilize the Ab-VEGF capture antibody (channels 3 and 5), the Ab-Ang1 antibody as a negative control (channels 2 and 4), and BSA as a passive control (channel 1). This strategy allowed us to set on-chip references that can be used to subtract any signal arising from non-specific binding events. The concentration of the solutions used during the incubation step was $20 \mu \mathrm{g} / \mathrm{mL}$ for Ab-VEGF/Ab-Ang1 and $10 \mathrm{mg} / \mathrm{mL}$ for BSA; the injected volume was $20 \mu \mathrm{L}$ for all solutions and immobilization lasted 1 hour. After removing the spotting tool, the biochips were rinsed with D-PBS and incubated overnight in a BSA solution in D-PBS $(10 \mathrm{mg} / \mathrm{mL})$ at $4{ }^{\circ} \mathrm{C}$, in order to block the whole surface. Fig. 3(a) shows a photograph of a biochip surface after the incubation step. Finally, the 1DPC surface is rinsed with D-PBS and the microfluidic cover is clicked on top (Fig. 1(a)) before inserting the complete biochip in the platform (Fig. S6, Supplementary Material).

Immunodetection fluorescence control assays, carried out with a different antigen (Angiopoietin 2), showed unaltered activity of the capture antibodies on the chip surface for more than a month, when the capture antibody-conjugated chips were stored dry at $4{ }^{\circ} \mathrm{C}$ (not reported here). We believe that similar stability, being related to the quality of the surface functionalization, can be assumed for anti-VEGF immobilized on the same biochips with the same protocol.

\subsection{Detection assay}

The VEGF detection assay implemented on the BSW platform involves a two-step procedure to achieve both label-free and fluorescence detection. Prior to each experiment, $1 \mathrm{~mL}$ of glycine- $\mathrm{HCl}$ (20 mM, pH 2.5) is injected and recirculated for 10 minutes in order to clean the biochip surface. Then the biochip is filled with D-PBS and the assay starts in the label-free detection mode, in which the angular position of the BSW resonance dip is continuously tracked.

Fig. 3(b) shows a CCD camera image acquired in label-free mode in D-PBS. Each column corresponds to one angular component of the reflected beam (Fig. 3(c)) and each row corresponds to one position along the illuminated region on the biochip surface. The BSW resonance appears as a dark vertical band. The large angular displacement of the resonance is evident at the regions 
where the five sensitive spots are situated, since the PDMS walls of the spotting tool leave residuals between adjacent channels that are detected in label-free mode. However, such PDMS markers do not perturb the measurements in any way.

After measuring a baseline in D-PBS, which is stable in a 15 min time interval thus demonstrating the stability of the chemically functionalized chip surface (Supplementary Material, Fig. S8), we injected and recirculated $400 \mu \mathrm{L}$ of the test solution for 10 minutes and followed the shift of the BSW dip. The injection and recirculation flow rates used in all the measurements was $30 \mu \mathrm{l} / \mathrm{min}$. The biochip was then thoroughly rinsed with D-PBS to complete the label-free detection. The overall label-free response is the shift of the BSW resonance angle with respect to the baseline level, following sample injection and rinsing in D-PBS.

The fluorescence assay is performed at the end of the label-free step. In the first phase, we injected and recirculated for 10 minutes a D-PBS solution of a biotinylated anti-VEGF detection antibody (Ab-VEGF*, $400 \mu \mathrm{L}, 1 \mu \mathrm{g} / \mathrm{mL}$ ). After rinsing with D-PBS, we recorded the background fluorescence signal. Then, we injected and recirculated for 10 minutes a D-PBS solution of a NeutrAvidin Protein DyLight $650(400 \mu \mathrm{L}, 1 \mu \mathrm{g} / \mathrm{mL})$. Finally, after washing with D-PBS, the fluorescence signal is recorded.

Fig. 3(d) displays the fluorescence detected by the CCD at the end of a typical assay in which VEGF was successfully detected. In order to distinguish the spots, we refer to the label-free map, which is aligned to the fluorescence map as both modes are imaged by the same detection optical system. Fig. 3(d) demonstrates that we can detect a clear fluorescence signal corresponding to spots 3 and 5, i.e. those functionalized with the Ab-VEGF capture antibody, and no fluorescence signal corresponding to spots 1 ((BSA) and 2, 4 (Ab-Ang1) used for referencing. 

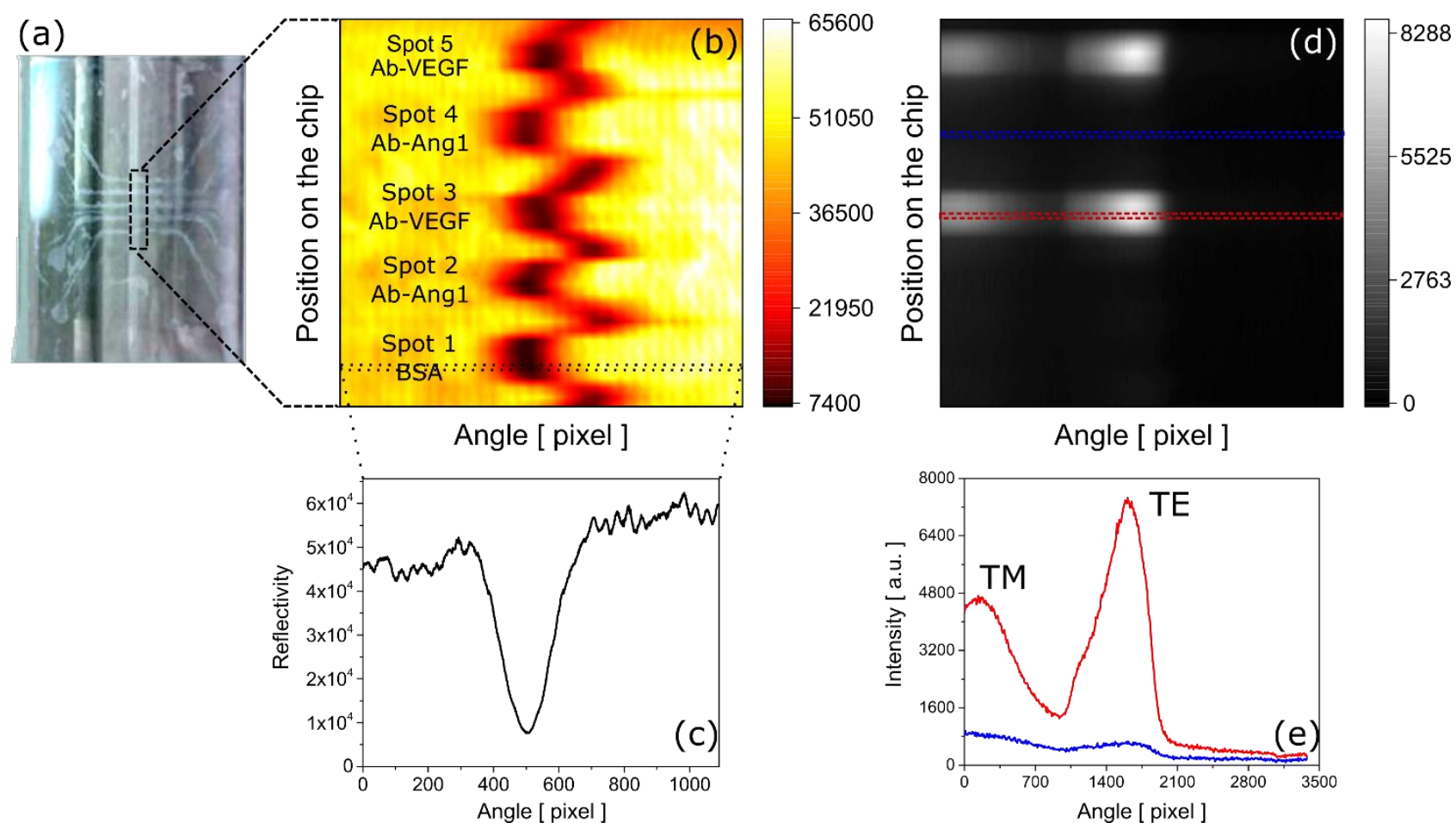

Angle [ pixel ]

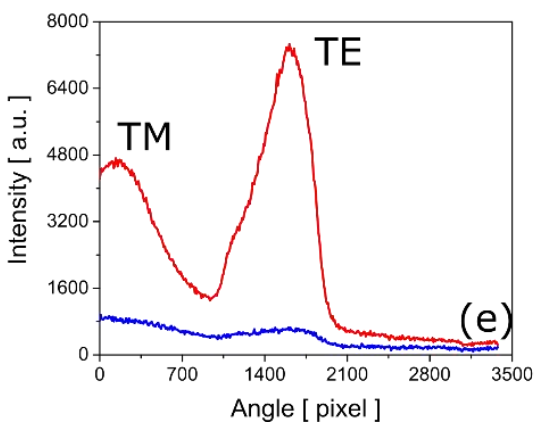

Fig. 3: (a) Picture of the 1DPC surface after the incubation with the spotting tool and before starting the experiment; (b) CCD reflectance map in label-free mode and (c) reflectance profile along the highlighted measurement line, the image was taken at the start of the experiment in D-PBS; (d) fluorescence emission recorded by the CCD camera at the end of an assay where VEGF was detected; (e) angular emission profiles of the highlighted regions.

For each spot, we acquired the angularly dispersed fluorescence emission in 50 adjacent CCD camera rows (see the region between dashed lines in Fig. 3(d)). The label-free map is used as a digital mask to integrate the fluorescence intensity of all lines within a single spot. First we subtract the background fluorescence recorded after the injection of the detection antibody, then the angular intensity distribution recorded in each CCD row (Fig. 3(e)) is integrated along the angle (horizontal direction in Fig. 3(d)). Hence, the integral values are averaged over the 50 rows and the average integrated intensity is assigned to each spot as a representative measured fluorescence value, together with the corresponding standard deviation of the 50 rows as uncertainty estimation.

\section{RESULTS AND DISCUSSION}

We tested more than 30 biochips for the detection of VEGF in different matrices. The first experiments and the calibration of the biosensing platform were done using solutions of VEGF in D-PBS. In the case of matrices with a higher level of complexity (cell supernatant and plasma 
samples), the label-free signal is affected by the critical problem of protein fouling. However, the experiments will show that the fluorescence detection mode is less affected by fouling and more sensitive. All experiments were carried out in duplicate.

\subsection{Label-free and fluorescence calibration curves in D-PBS}

Fig. 4 shows the results of the label-free calibration assays carried out with three identical biochips and for three different known concentrations of VEGF spiked in D-PBS (50, 250, $500 \mathrm{ng} / \mathrm{mL})$. At such concentrations, the sensograms show a clear difference between the angular shift recorded in the signal and reference spots. The lowest concentration $(50 \mathrm{ng} / \mathrm{mL})$ can be clearly detected. The residual shifts after the D-PBS washing were averaged in the signal and reference spots (50 rows). The label-free sensor response is then calculated as the difference between such averaged values. The results are reported in Fig. 4(d). The label-free response is linear and the sensitivity is evaluated as $S_{\mathrm{LF}}=(0.0286 \pm 0.0014)$ pixel / $[\mathrm{ng} / \mathrm{mL}]$. The standard deviation of the noise, calculated on a 5 minute baseline, is $\sigma_{\mathrm{LF}}=0.157$ pixel. When the data shown in Fig. 4 are analyzed according to procedures that are commonly reported in literature (Wang et al., 2009), one can evaluate the limit of detection in the label-free mode as $\mathrm{LoD}_{\mathrm{LF}}=2 \sigma_{\mathrm{LF}} / \mathrm{S}_{\mathrm{LF}}=(11.5 \pm 0.5) \mathrm{ng} / \mathrm{mL}$. The LoD $\mathrm{LF}_{\mathrm{LF}}$ can be expressed in terms of the minimum mass coverage detectable in the label free mode $\Gamma_{L F}^{M I N}$ by making use of the following expression (Sinibaldi et al., 2015b):

$$
\Gamma_{L F}^{M I N}=\frac{\Delta \theta_{M I N} L_{p e n}}{S_{V} \partial n / \partial C}
$$

where $\Delta \theta_{\mathrm{MIN}}=2 \sigma_{\mathrm{LF}}=0.314$ pixel, $\mathrm{L}_{\mathrm{pen}}=114 \mathrm{~nm}$ is the length of the $\mathrm{BSW}$ evanescent tail in the external medium for the present $1 \mathrm{DPC}$ design, $\mathrm{S}_{\mathrm{V}}=4.8 \times 10^{4} \mathrm{pixel} / \mathrm{RIU}$ is the measured sensitivity of the BSW resonance position with respect to variations of the refractive index of the external medium (RIU is refractive index units) and $\partial \mathrm{n} / \partial \mathrm{C}$ is the refractive index increment of the molecules and $\partial \mathrm{n} / \partial \mathrm{C}=0.19 \mathrm{~cm}^{3} / \mathrm{g}$ for most of proteins (Voros, 2004). We get $\Gamma_{L F}^{M I N}=3.9 \mathrm{pg} / \mathrm{mm}^{2}$.

Despite the LoD $\mathrm{LF}$ (and $\Gamma_{L F}^{M I N}$ ) is in the range of other optical label-free platforms making use of surface waves, it is still larger than previously reported plasma VEGF concentrations in healthy humans and cancer patients, ranging from a few tens to a few hundreds of pg/ml (Iinuma, 2010; Jelkmann, 2001; Ławicki et al., 2016). 

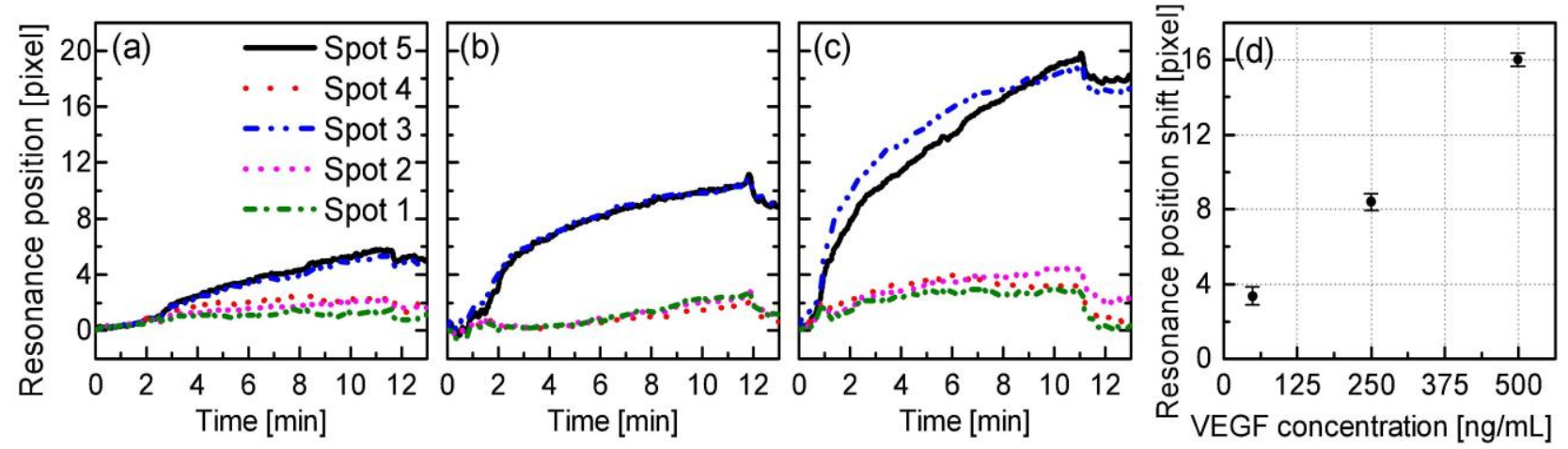

Fig. 4: Label-free sensorgrams for the detection of VEGF in D-PBS at concentrations of (a) 50, (b) 250 and (c) $500 \mathrm{ng} / \mathrm{mL}$. Spots 3 and 5 have been functionalized with VEGF specific antibody; while Spots 2, 4 and 1 are reference spots. (d) Resonance position shift plotted against biomarker concentration shows a good linearity.

We calibrated the operation in the fluorescence detection mode by setting the minimum concentration measured in label-free mode as the largest for fluorescence. Therefore, VEGF was spiked in D-PBS at known concentrations in the range $2.5-50 \mathrm{ng} / \mathrm{mL}$. Fig. 5 presents the background subtracted integrated fluorescence intensities recorded in different assays carried out with six biochips, which were prepared identically. The error bars represent the standard deviation of the integral values.

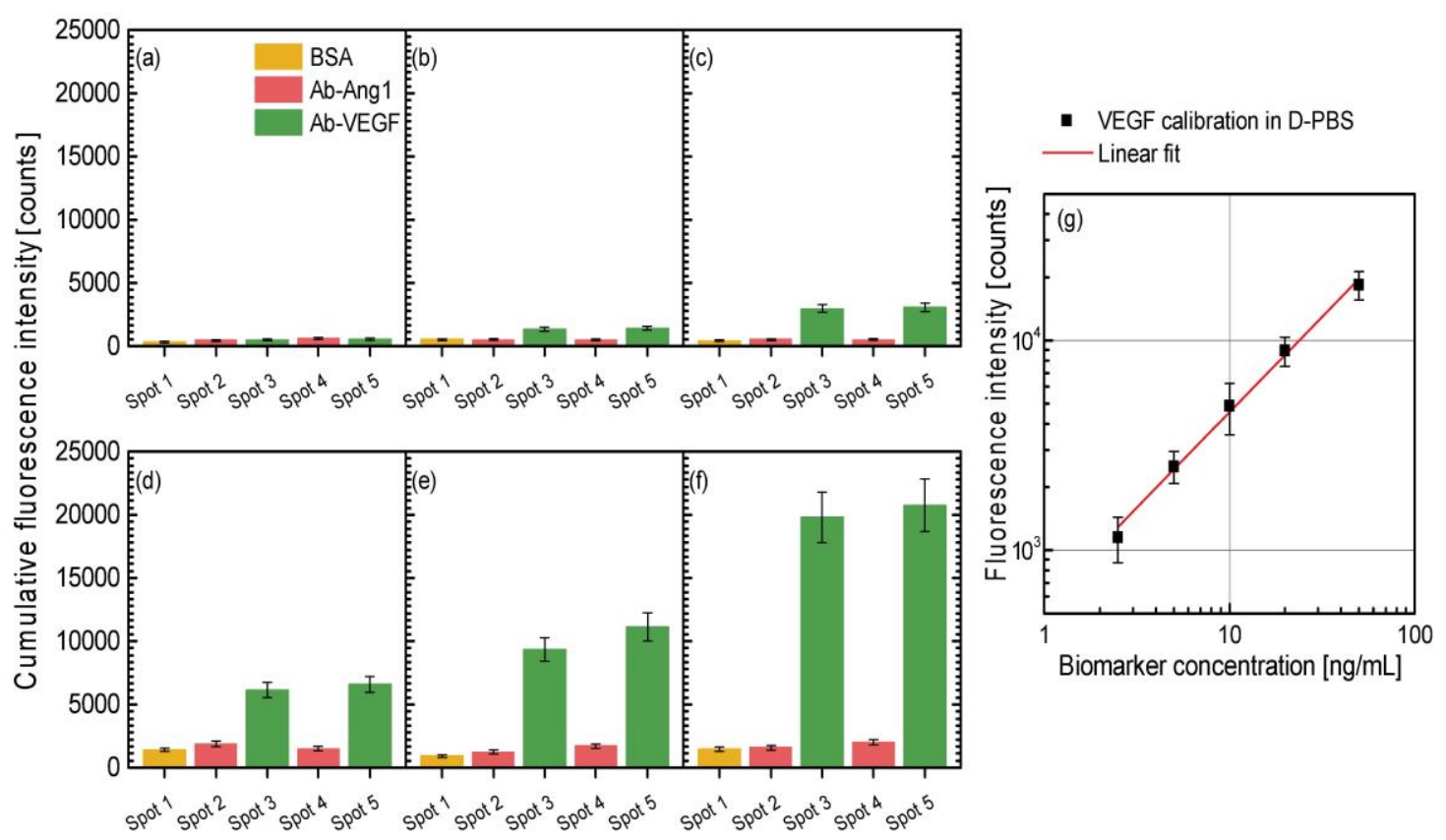


Fig. 5: Fluorescence intensity from VEGF solutions at different concentrations in D-PBS. (a) 0; (b) 2.5, (c) 5 , (d) 10 , (e) 20 , (f) $50 \mathrm{ng} / \mathrm{mL}$. (g) Calibration curve obtained after subtraction of the averaged reference Spots intensity to the averaged signal spots.

Fig. 5(a) shows the case in which no VEGF was injected in the biochip (the buffer only). It appears that the detection antibody is non-specifically adsorbed onto the sensor surface and Neutravidin can bind to it, resulting in a non-specific fluorescence signal. The signal obtained is uniform over the whole biochip's surface. Fig. 5(b-f) refer to the case in which solutions containing VEGF were injected, with increasing concentration. Each graph corresponds to a different experiment performed with a fresh biochip, indicating reproducibility of the assays. Increasing fluorescence intensities are apparent in the signal spots, exhibiting a good contrast with respect to the reference ones. For each VEGF concentration, the final fluorescence intensity is estimated as the difference between the averaged intensities of the spots 3 and 5 and the reference. The corresponding variances are summed in order to obtain the final standard deviations. The lowest concentration (2.5 $\mathrm{ng} / \mathrm{mL}$ ) can be clearly detected in the fluorescence mode.

In Fig. 5(g) we show the resulting calibration curve. Fluorescence intensities are linearly fitted, resulting in a sensitivity of $S_{F}=(460 \pm 26)$ counts $/[\mathrm{ng} / \mathrm{mL}]$. Similar to the label-free mode, making use of the standard deviation of the smallest concentration measured ( $\sigma_{\mathrm{F}}=150$ counts), one can evaluate the limit of detection for the fluorescence mode as $\operatorname{LoD}_{\mathrm{F}}=(0.65 \pm 0.04) \mathrm{ng} / \mathrm{mL}$. Such value is one order of magnitude lower than the $\mathrm{LoD}_{\mathrm{LF}}$ reported above for label-free operation, and it is closer to the clinically relevant VEGF concentration in human plasma samples. Assuming a linear dependence of mass coverage on the VEGF concentration, which is valid between LoD $\mathrm{LF}_{\text {and }}$

LOD $\mathrm{F}$, we estimate $\Gamma_{F}^{M I N}=\frac{\operatorname{LoD}_{F}}{\operatorname{LoD}_{L F}} \Gamma_{L F}^{M I N}=0.2 \mathrm{pg} / \mathrm{mm}^{2}$. It is worth mentioning that the standard deviation associated to the fluorescence intensity signal is correlated to the uniformity of the capture antibodies' surface density on each spot obtained during the bio-conjugation step. The calibration curve shown in Fig. 5(g) was used to evaluate the unknown VEGF concentration in cell culture supernatant and spiked plasma samples.

\subsection{Measurement of supernatant samples}

The VEGF concentration in cell supernatants was preliminary assessed via ELISA (Fig. S1, Supplementary Material). Wild-type MCF-7 cells expressed a VEGF basal level equal to $77 \mathrm{pg} / \mathrm{mL}$, while VEGF $_{165}$-overexpressing MCF-7 released an increased amount equal to $15.5 \mathrm{ng} / \mathrm{mL}$. To test 
the platform, we used undiluted (Fig. 6(a)) and D-PBS diluted (ratio 1:4) cell supernatants (Fig. 6(b)).

From the differential fluorescence intensities evaluated from Fig. 6 (a-b) and making use of the calibration curve (Fig. 5(g)) we estimated the VEGF concentration: $(14.0 \pm 2.4) \mathrm{ng} / \mathrm{mL}$ and $(4.0 \pm 0.8) \mathrm{ng} / \mathrm{mL}$ for the undiluted and diluted samples, respectively. In Fig. 6(c) we plot the fluorescence calibration curve together with data points corresponding to the intensities for the cell culture supernatant samples. The plot shows a good agreement between our platform and the results obtained by ELISA, demonstrating the accuracy of the method. The intensities obtained from the diluted samples are consistent with the undiluted ones and the deviations among the measured spots in supernatants are within the uncertainty of the calibration curve. The reported results demonstrate that our biosensing platform can successfully perform biomarker detection in cells culture supernatants.
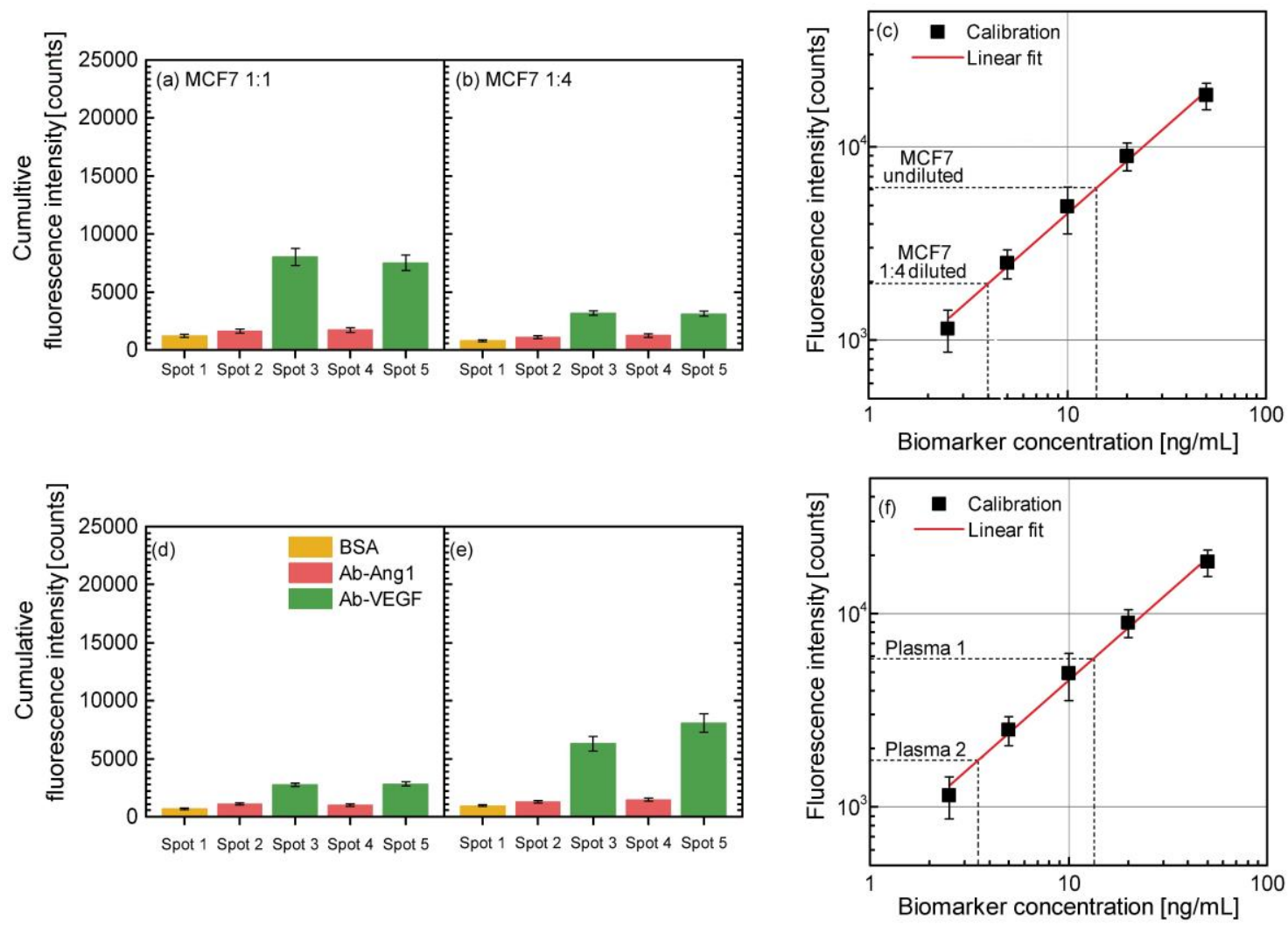

Fig. 6: (a-c) Fluorescence intensities from cell culture supernatant samples: (a) undiluted sample of VEGF $_{165}$-overexpressing MCF-7 cells containing VEGF at $15.5 \mathrm{ng} / \mathrm{mL}$; (b) 1:4 diluted sample of VEGF $_{165}$-overexpressing MCF-7 cells. (c) Calibration curve (Fig. 5(g)) and cell culture supernatant results. (d-f) Fluorescence measurements of plasma samples with a spiked VEGF concentration of 
(d) $3.75 \mathrm{ng} / \mathrm{mL}$ (Plasma 2) and (e) $15 \mathrm{ng} / \mathrm{mL}$ (Plasma 1). (f) Calibration curve and spiked plasma samples results. The calibration curves in (c) and (f) are taken from Fig. 5(g).

\subsection{Measurement of spiked blood samples}

Using ELISA, the VEGF concentration in human plasma samples was measured to be in the range of 17-301 pg/ml (Fig. S2, Supplementary Material). The sample volume used in each measurement carried out with the BSW platform was $300 \mu \mathrm{l}$.

All human plasma samples in this study show a VEGF concentration below the $\mathrm{LoD}_{\mathrm{F}}$ of the sensing platform. Therefore, in order to test our platform with a complex matrix, we spiked a certain amount of VEGF in two plasma samples to obtain two samples at concentration 3.75 and $15 \mathrm{ng} / \mathrm{mL}$. The results of the assays performed in fluorescence mode are shown in Fig. 6(d-f). The fluorescence signals in the spots 3 and 5 can be well distinguished from those in the reference spots. The fluorescence intensities return, through the calibration curve, VEGF concentrations of $(3.5 \pm 0.7) \mathrm{ng} / \mathrm{mL}$ and $(14.0 \pm 2.4) \mathrm{ng} / \mathrm{mL}$, for the two samples. Such values are in good agreement with the spiked concentrations. It is worth mentioning that protein fouling does not affect the fluorescence operation mode, both for supernatant and plasma samples from different donors. The results presented in Fig. 6(d-f) show that the platform enables determination of different biomarker concentrations in plasma matrix.

\section{CONCLUSIONS}

We reported the successful detection of VEGF cancer biomarker in a range of biological matrices (buffer solutions, cell supernatants, and human plasma) through the use of a BSW-based biosensing platform. The BSW platform takes advantage of a tandem label-free and fluorescence configuration. The label-free mode of operation is characterized by an estimated $\operatorname{LoD}_{\mathrm{LF}}$ of $(11.0 \pm 0.5) \mathrm{ng} / \mathrm{mL}$ and can provide useful information about the kinetics of the binding reactions. In the fluorescence mode of operation the platform detected VEGF molecules down to $3.5 \mathrm{ng} / \mathrm{mL}$ in human plasma samples, with an estimated $\mathrm{LoD}_{\mathrm{F}}$ of $0.65 \mathrm{ng} / \mathrm{mL}$, close to the plasma VEGF concentration values previously reported for cancer patients (i.e., in the order of one hundred or a few hundreds of $\mathrm{pg} / \mathrm{ml}$ ). The measurement is as simple as other immunoassays, requires a shorter time (30 minutes), has a good LoD and uses non-expensive disposable chips ready for mass production. In addition, the possibility of detecting multiple analytes in a multiplexing scheme is already feasible. 


\section{ACKNOWLEDGEMENTS}

This work was funded by the European Commission through the project BILOBA (Grant agreement 318035). M.A., L.N., F.B. kindly acknowledge Prof. Roy Bicknell (University of Birmingham) for giving the MCF-7 cells.

\section{REFERENCES}

Angelini, A., Barakat, E., Munzert, P., Boarino, L., De Leo, N., Enrico, E., Giorgis, F., Herzig, H.P., Pirri, C.F., Descrovi, E., 2014. Focusing and Extraction of Light mediated by Bloch Surface Waves. Sci. Rep. 4, 5428. doi:10.1038/srep05428

Angelini, A., Enrico, E., De Leo, N., Munzert, P., Boarino, L., Michelotti, F., Giorgis, F., Descrovi, E., 2013. Fluorescence diffraction assisted by Bloch surface waves on a one-dimensional photonic crystal. New J. Phys. 15, 73002. doi:10.1088/1367-2630/15/7/073002

Anopchenko, A., Occhicone, A., Rizzo, R., Sinibaldi, A., Figliozzi, G., Danz, N., Munzert, P., Michelotti, F., 2016. Effect of thickness disorder on the performance of photonic crystal surface wave sensors. Opt. Express 24, 7728. doi:10.1364/OE.24.007728

Badugu, R., Nowaczyk, K., Descrovi, E., Lakowicz, J.R., 2013. Radiative decay engineering 6: Fluorescence on one-dimensional photonic crystals. Anal. Biochem. 442, 83-96. doi:10.1016/j.ab.2013.07.021

Comşa, Ş., Cîmpean, A.M., Raica, M., 2015. The Story of MCF-7 Breast Cancer Cell Line: 40 years of Experience in Research. Anticancer Res. 35, 3147-54.

Cooper, M. a, 2002. Optical biosensors in drug discovery. Nat. Rev. Drug Discov. 1, 515-528. doi:10.1038/nrd838

Descrovi, E., Barakat, E., Angelini, A., Munzert, P., De Leo, N., Boarino, L., Giorgis, F., Herzig, H.P., 2013. Leakage radiation interference microscopy. Opt. Lett. 38, 3374-6. doi:10.1364/OL.38.003374

Descrovi, E., Sfez, T., Quaglio, M., Brunazzo, D., Dominici, L., Michelotti, F., Herzig, H.P., Martin, O.J.F., Giorgis, F., 2010. Guided bloch surface waves on ultrathin polymeric ridges. Nano Lett. 10, 2087-2091. doi:10.1021/nl100481q

Dey, B., Thukral, S., Krishnan, S., Chakrobarty, M., Gupta, S., Manghani, C., Rani, V., 2012. DNA-protein interactions: methods for detection and analysis. Mol. Cell. Biochem. 365, 279299. doi:10.1007/s11010-012-1269-z

Fan, X., White, I.M., Shopova, S.I., Zhu, H., Suter, J.D., Sun, Y., 2008. Sensitive optical biosensors for unlabeled targets: A review. Anal. Chim. Acta 620, 8-26. doi:10.1016/j.aca.2008.05.022

Fang, Y., Ferrie, A.M., Fontaine, N.H., Mauro, J., Balakrishnan, J., 2006. Resonant Waveguide Grating Biosensor for Living Cell Sensing. Biophys. J. 91, 1925-1940. doi:10.1529/biophysj.105.077818

Ferrara, N., 2010. Vascular endothelial growth factor and age-related macular degeneration: from basic science to therapy. Nat. Med. 16, 1107-1111. doi:10.1038/nm1010-1107

Fiorilli, S., Rivolo, P., Descrovi, E., Ricciardi, C., Pasquardini, L., Lunelli, L., Vanzetti, L., Pederzolli, C., Onida, B., Garrone, E., 2008. Vapor-phase self-assembled monolayers of aminosilane on plasma-activated silicon substrates. J. Colloid Interface Sci. 321, 235-241. doi:10.1016/j.jcis.2007.12.041

Guo, X., 2012. Surface plasmon resonance based biosensor technique: A review. J. Biophotonics 5, 483-501. doi:10.1002/jbio.201200015

Iinuma, 2010. Association of plasma VEGF-A, soluble VEGFR-1 and VEGFR-2 levels and clinical response and survival in advanced colorectal cancer patients receiving bevacizumab with 
modified FOLFOX6. Oncol. Lett. 1, 253-259. doi:10.3892/ol_00000045

Inan, Hakan; Poyraz, Muhammet; Inci, Fatih; Lifson, Mark A.; Baday, Murat; Cunningham, Brian T.; Demirci, U., 2017. Photonic crystals : emerging biosensors and their promise for point-ofcare applications. Chem. Soc. Rev. 46, 366-388. doi:10.1039/C6CS00206D

Jelkmann, W., 2001. Pitfalls in the measurement of circulating vascular endothelial growth factor. Clin. Chem. 47, 617-23.

Konopsky, V., Karakouz, T., Alieva, E., Vicario, C., Sekatskii, S., Dietler, G., 2013. Photonic Crystal Biosensor Based on Optical Surface Waves. Sensors 13, 2566-2578. doi:10.3390/s130202566

Ławicki, S., Zajkowska, M., Głażewska, E.K., Będkowska, G.E., Szmitkowski, M., 2016. Plasma levels and diagnostic utility of VEGF, MMP-9, and TIMP-1 in the diagnosis of patients with breast cancer. Onco. Targets. Ther. 9, 911-9. doi:10.2147/OTT.S99959

Ligler, F.S., Taitt, C.A.R., 2008. Optical biosensors : today and tomorrow. Elsevier.

Mathias, P.C., Ganesh, N., Chan, L.L., Cunningham, B.T., 2007. Combined enhanced fluorescence and label-free biomolecular detection with a photonic crystal surface. Appl. Opt. 46, 2351. doi:10.1364/AO.46.002351

Pepe, M.S., Etzioni, R., Feng, Z., Potter, J.D., Thompson, M.L., Thornquist, M., Winget, M., Yasui, Y., 2001. Phases of biomarker development for early detection of cancer. J. Natl. Cancer Inst. 93, 1054-61. doi:11459866

Ray, K., Badugu, R., Lakowicz, J.R., 2015. Bloch surface wave-coupled emission from quantum dots by ensemble and single molecule spectroscopy. RSC Adv. 5, 54403-54411. doi:10.1039/C5RA03413B

Ricciardi, S., Frascella, F., Angelini, A., Lamberti, A., Munzert, P., Boarino, L., Rizzo, R., Tommasi, A., Descrovi, E., 2015. Optofluidic chip for surface wave-based fluorescence sensing. Sensors Actuators, B Chem. 215, 225-230. doi:10.1016/j.snb.2015.03.063

Rivolo, P., Michelotti, F., Frascella, F., Digregorio, G., Mandracci, P., Dominici, L., Giorgis, F., Descrovi, E., 2012. Real time secondary antibody detection by means of silicon-based multilayers sustaining Bloch surface waves. Sensors Actuators, B Chem. 161, 1046-1052. doi:10.1016/j.snb.2011.12.006

Rizzo, R., Danz, N., Michelotti, F., Maillart, E., Anopchenko, A., Wächter, C., 2014. Optimization of angularly resolved Bloch surface wave biosensors. Opt. Express 22, 23202. doi:10.1364/OE.22.023202

Shinn, M., Robertson, W.M., 2005. Surface plasmon-like sensor based on surface electromagnetic waves in a photonic band-gap material. Sensors Actuators B Chem. 105, 360-364. doi:10.1016/j.snb.2004.06.024

Sikarwar, B., Singh, V. V., Sharma, P.K., Kumar, A., Thavaselvam, D., Boopathi, M., Singh, B., Jaiswal, Y.K., 2017. DNA-probe-target interaction based detection of Brucella melitensis by using surface plasmon resonance. Biosens. Bioelectron. 87, 964-969. doi:10.1016/j.bios.2016.09.063

Sinibaldi, A., Anopchenko, A., Rizzo, R., Danz, N., Munzert, P., Rivolo, P., Frascella, F., Ricciardi, S., Michelotti, F., 2015a. Angularly resolved ellipsometric optical biosensing by means of Bloch surface waves. Anal. Bioanal. Chem. 407, 3965-3974. doi:10.1007/s00216-015-8591-8

Sinibaldi, A., Danz, N., Anopchenko, A., Munzert, P., Schmieder, S., Chandrawati, R., Rizzo, R., Rana, S., Sonntag, F., Occhicone, A., Napione, L., De Panfilis, S., Stevens, M.M., Michelotti, F., 2015b. Label-Free Detection of Tumor Angiogenesis Biomarker Angiopoietin 2 Using Bloch Surface Waves on One Dimensional Photonic Crystals. J. Light. Technol. 33, 33853393. doi:10.1109/JLT.2015.2448795

Sinibaldi, A., Danz, N., Descrovi, E., Munzert, P., Schulz, U., Sonntag, F., Dominici, L., Michelotti, F., 2012. Direct comparison of the performance of Bloch surface wave and surface plasmon polariton sensors. Sensors Actuators, B Chem. 174, 292-298. doi:10.1016/j.snb.2012.07.015 
Tan, W., Sabet, L., Li, Y., Yu, T., Klokkevold, P.R., Wong, D.T., Ho, C.-M., 2008. Optical protein sensor for detecting cancer markers in saliva. Biosens. Bioelectron. 24, 266-271. doi:10.1016/j.bios.2008.03.037

Tighe, P.J., Ryder, R.R., Todd, I., Fairclough, L.C., 2015. ELISA in the multiplex era: Potentials and pitfalls. Proteomics - Clin. Appl. doi:10.1002/prca.201400130

Ulber, R., Frerichs, J.-G., Beutel, S., 2003. Optical sensor systems for bioprocess monitoring. Anal. Bioanal. Chem. 376, 342-8. doi:10.1007/s00216-003-1930-1

Voros, J., 2004. The Density and Refractive Index of Adsorbing Protein Layers. Biophys. J. 87, 553-561 The. doi:10.1529/biophysj.103.030072

Walker, J.M., 2009. Biosensors and Biodetection, Biosensors, Methods in Molecular Biology. Humana Press, Totowa, NJ. doi:10.1007/978-1-60327-567-5

Zhao, Y., Zhao, X., Gu, Z., 2010. Photonic Crystals in Bioassays. Adv. Funct. Mater. 20, 29702988. doi:10.1002/adfm.201000098 